\title{
Discordar a través de la comida. Descendientes de chinos y nuevos inmigrantes en Tapachula, Chiapas
}

\section{Discording through food. Chinese descendants and new immigrants in Tapachula, Chiapas}

\author{
MIGUEL LISBONA GUILLÉN \\ Universidad Nacional Autónoma de México, \\ Ciudad de México, México \\ mlisbona@unam.mx
}

\section{ULISES ALBERTO RINCÓN ZÁRATE \\ Universidad Autónoma de Chiapas, \\ Tuxtla Gutiérrez, Chiapas, México \\ narz@hotmail.com}

Resumen: En la capital de la costa de Chiapas (México), Tapachula, la llegada de nuevos inmigrantes chinos durante el actual siglo para abrir negocios de restauración coincide con la presencia de similares negocios regentados por descendientes de la histórica inmigración china a la región producida, principalmente, a principios del siglo XX. A través del trabajo efectuado por más de una década, y mediante la observación participante y entrevistas, el artículo muestra cómo las representaciones sociales en torno a la comida ofrecida en los negocios de la ciudad establecen una clara diferenciación entre los históricos y nuevos negocios gastronómicos. Esta disociación, en este caso, cuestiona los discursos homogéneos sobre 
la identidad y los referentes territoriales y nacionales que la construyen. Así, la comida china se legitima por originarse en una herencia histórica y familiar de los descendientes de chinos, y se degrada cuando es aportada por los nuevos inmigrantes recién arribados a Tapachula.

Palabras clave: comida china, restauración, inmigración, identidad, conflicto.

Abstract: In the capital of the coast of Chiapas (Mexico), Tapachula, the arrival of new Chinese immigrants, during the current century, to open restaurant businesses coincides with the presence of similar businesses run by descendants of the historical Chinese immigration to the region. This immigration occurred mainly at the beginning of the 20th century. Through the work carried out for more than a decade, and by means of participant observation and interviews, the article shows how the social representations around the food offered in the city's businesses establish a clear differentiation between the historical and new gastronomic businesses. Dissociation, in this case, that questions the homogeneous discourses on identity and the territorial and national referents that construct it. Thus, Chinese food is legitimized because it originates in a historical and family heritage of Chinese descendants, and is degraded when it is brought by new immigrants who have recently arrived in Tapachula.

Keywords: chinese food, restoration, inmigration, identity, conflict

Citar como: Lisbona-Guillén, M., \& Rincón-Zárate, U. A. (2022). Discordar a través de la comida. Descendientes de chinos y nuevos inmigrantes en Tapachula, Chiapas. Revista Internacional de Estudios Asiáticos, 1(1), 2-29.

Fecha de recepción: 27-08-2021 | Fecha de aceptación: 13-10-2021 


\section{Introducción}

Una de las características de la región costera de Chiapas, con especial relevancia en su principal ciudad, Tapachula, ha sido la incorporación de la comida china como patrimonio cultural regional. Los inmigrantes chinos de principios del siglo XX, y en especial sus descendientes, han establecido negocios gastronómicos en la región. No es, por supuesto, el único rubro comercial o profesional al que se dedican los descendientes de chinos, sin embargo, se ha convertido en el más reconocido. Por ello, sin llegar a recordar los enclaves étnicos, como ocurre en otros lugares del planeta, ${ }^{1}$ en la actualidad la presencia de restaurantes chinos es visible en Tapachula, al mismo tiempo que la denominada comida china es referente identitario local para su población, aunque no tengan nexos consanguíneos con dicha inmigración.

Lo anterior ha posibilitado hablar de una identidad regional más abierta y cosmopolita, o al menos discursivamente así se expresa, donde elementos procedentes del exterior, de los aportes culturales producto de la inmigración, se hacen propios y se incorporan al discurso de la tradición local; ${ }^{2}$ un hecho producido gracias a inmigraciones históricas de chinos, japoneses, alemanes o españoles en la misma región costera. Sin embargo, tal supuesto cosmopolitismo, tangible en la gastronomía, se ha visto interpelado social y discursivamente tras el arribo de nuevos inmigrantes chinos a Tapachula.

De este modo, la comida servida en los restaurantes ya considerados tradicionales en la actualidad ha identificado lo chino en Chiapas. Esta identificación se basa en preceptos culturales defendidos ahora por los descendientes de chinos, donde destacan las danzas y, sobre todo, la gastronomía. Ello contrasta con los nexos transnacionales de los nuevos in-

1 Jesús Tébar Arjona, "Patrones espaciales de la diáspora china en el mundo, España y Madrid”, Historia Actual Online, no. 30 (2013): 91-92.

2 Miguel Lisbona Guillén, Alli donde lleguen las olas del mar. Pasado y presente de los chinos en Chiapas (México: UNAM-PROIMMSE/ CONACULTA-CONECULTA, 2014). 
migrantes chinos que han llegado a la costa chiapaneca en los últimos cuatro lustros, pues se trata de personas que mantienen vínculos en distintos países, además de tener experiencias migratorias antes de asentarse en Tapachula. Su oferta gastronómica es otra, en negocios de comida rápida. Si la comida china parecía un valor intocable, diacrítico de identificación de los descendientes de chinos, a la vez que significaba una aportación a la identidad regional legitimada por su carácter tradicional, en la actualidad se convierte en un elemento de debate por cuestionarse su esencialidad en los procesos de circulación cultural global. ${ }^{3}$

Por otra parte, la supuesta cohesión de la colonia china en la región a principios del siglo XX, manifestada a través de agrupaciones como el Partido Nacionalista Chino (Kuo Min Tang) y, posteriormente, por la Asociación China del Soconusco A.C., no ha tenido continuidad en el tiempo debido a las dificultades para mantener la vigencia de la última asociación. Esta situación intenta ser subsanada por varios descendientes de chinos que están pugnando por revitalizar la asociación y recuperar la casa que albergó al Kuo Min Tang. Si estas formas de organización, o de imaginarse como una comunidad, permitieron generar un cierto sentido de identidad china entre los inmigrantes, no es menos cierto que lo que prevaleció entre losllegados de Asia fue una progresiva integración a la sociedad de acogida; proceso que facilitó la asunción de ciertos elementos culturales chinos como representativos de la identidad local. Esta integración fue facilitada por los matrimonios con mujeres mexicanas, al ser los inmigrantes en su mayoría varones, y por las relaciones establecidas con las élites políticas locales desde las asociaciones creadas por los chinos.

Esa realidad es rebatida cuando se produce la llegada de una nueva oleada de inmigrantes durante el presente siglo. La situación coincide con el esfuerzo de las asociaciones chinas por tener una mayor visibilidad regional e internacional. ${ }^{4}$ Estas iniciativas para incorporarse a las conexiones

3 Ulrich Beck, ¿Qué es la globalización? Falacias del globalismo, respuestas a la globalización (Lanús: Paidós, 2008).

4 Comunidad China del Soconusco A.C. (Tapachula) y Comunidad China de Escuintla (Escuintla). 
transnacionales, ${ }^{5}$ especialmente en temas económicos y educativos, buscan beneficiar a sus miembros pero, en especial, refuerzan la influencia política de algunos de sus miembros.

Los nuevos inmigrantes, quienes no suelen superar la cuarentena de años, no toman Tapachula como un destino definitivo para establecerse. Tampoco tienen un único origen, aunque algunos de ellos refieren como procedencia la provincia de Cantón. La mayoría son trabajadores asalariados de los propietarios de los negocios y tanto dueños como trabajadores no suelen involucrarse en actividades sociales más allá de su espacio doméstico y laboral. Estos recién llegados aportan otra oferta gastronómica para antagonizar consumos y, también, cuestionar identificaciones nacionales. Su presencia ha significado una confrontación entre los descendientes de inmigrantes de larga data y los recién llegados a través de la comida china. Esta disputa sitúa en el centro del debate la identidad nacional china y se refleja en la propia dinámica cultural de la capital del Soconusco. Una discusión que, con distintos matices, ya se había producido en el Soconusco a través de las diferencias establecidas entre distintas organizaciones dedicadas a las danzas del dragón y del león en municipios del Soconusco. ${ }^{6}$

El ficticio parentesco propiciado por los Estados modernos y la conformación de su nación, ${ }^{7}$ no encajan en procesos como el chiapaneco porque los sentidos de pertenencia y reconocimiento de los descendientes de chi-

5 Nina Glick Schiller, "Transmigrants and Nation States: Something Old and Something New in the U.S. Immigrant Experience", en Handbook of International Migration: The American Experience, ed. por C. Hirschman, P. Kasinitz y J. De Wind (Nueva York: Russell Sage Foundation, 1999), 94-119. Alejandro Portes, Cristina Escobar y Alexandria Walton. "Organizaciones transnacionales de inmigrantes y desarrollo: un estudio comparativo". Migración y Desarrollo 6 (2006): 3-44.

6 Miguel Lisbona Guillén, "Danzas como tradición y como disputa: la ilusión comunitaria china en el Soconusco chiapaneco". Peninsula X, no. 1 (2015): 9-28. 7 Benedict Anderson, Comunidades imaginadas. Reflexiones sobre el origen y la difusión del nacionalismo (México: Fondo de Cultura Económica, 1993). 
nos en el Soconusco contrastan con los de los recién llegados. Su llegada propicia repensar el discurso sobre lo chino a través de una comida dotada de una biografía cultural por parte de los descendientes de chinos y que refiere con términos prejuiciosos a la ofrecida por los nuevos inmigrantes.

Los históricos restaurantes de los descendientes de chinos brindan, desde su perspectiva, un producto distintivo gracias a la condición patrimonial creada a su alrededor, a la par que acceder a él implica para sus consumidores contar con recursos económicos que evidencian una distinción de clase. Así, la comida china elaborada y vendida por los descendientes de los primeros inmigrantes se erige en diacrítico ${ }^{8}$ social entre la ciudadanía tapachulteca. Por el contrario, los nuevos restaurantes chinos han creado sus establecimientos, muy visibles alrededor del centro de la ciudad, con comida rápida, express, o con la modalidad de restaurante tipo buffet, lo cual se ha extendido a otras ciudades chiapanecas como San Cristóbal de Las Casas ${ }^{9}$ y la capital estatal, Tuxtla Gutiérrez.

Con el arribo de chinos en el presente siglo, su competencia comercial pone en juego elementos tangibles y simbólicos a la hora de diferenciar la oferta gastronómica en Tapachula. El mismo origen nacional se cuestiona a través de discursos sobre la ancestralidad y la autenticidad de las recetas familiares de los primeros inmigrantes; lo cual constituye un valor agregado para distinguirse como restaurante de alta cocina china en la ciudad. Frente a ello, los nuevos restaurantes son descalificados por carecer de elementos de arraigo y pertenencia local, una calificación que también los ubica dentro de una cocina china baja o popular. Esta última circuns-

8 Pierre Bourdieu, La distinción. Criterio y bases sociales del gusto (México: Taurus, 1988). Ulises Rincón Zárate, "El posicionamiento socio-espacial de los descendientes de inmigrantes chinos y japoneses en Tapachula, Chiapas” (Tesis de Maestría, Centro de Investigaciones y Estudios Superiores en Antropología Social, 2019).

9 María del Jazmín Hernández López, "El comercio chino en San Cristóbal de Las Casas, Chiapas: tendencias hacia la empresa familiar, el empresariado étnico y el sistema de redes familiares" (Tesis de Maestría en Antropología Social, Centro de Investigaciones y Estudios Superiores en Antropología Social, 2015). 
tancia manifiesta varios aspectos de relevancia y que se apuntarán en este artículo: por una parte, la utilización de la gastronomía para establecer diferencias culturales entre los descendientes de chinos y los inmigrantes de nuevo arribo; por otra, exterioriza las diferenciaciones sociales palpables en Tapachula y que tienen en la comida china el elemento de identificación patrimonial de la ciudad chiapaneca. ${ }^{10} \mathrm{~A}$ esos dos perceptibles aspectos hay que añadir otra distinción a seguir en los próximos años sobre la consideración sanguínea, biológica, de las diferencias entre ambos colectivos y donde los nuevos inmigrantes muestran recelos sobre la autenticidad china de los descendientes de la primera migración china a Chiapas.

En este texto se hará referencia al marketing gastronómico construido en torno a la comida china en Tapachula y las distinciones generadas en la preparación y consumo de platillos chinos en la ciudad. Para ese cometido se emplean distintas perspectivas que incluyen los conceptos de biografía cultural de Kopytoff, ${ }^{11}$ los procesos de globalización cultural de la gastronomía china, ${ }^{12}$ y el marketing de experiencias memorables. ${ }^{13} \mathrm{La}$ mercantilización de productos homogenizados en su proceso de producción

10 Rincón Zárate, "El posicionamiento socio-espacial”.

11 Igor Kopytoff, "La biografía cultural de las cosas: La mercantilización como proceso", en La vida social de las cosas. Perspectiva cultural de las mercancias, ed. por Arjun Appadurai (México: CONACULTA/Editorial Grijalbo, 1991), 89124.

12 Richard R. Wilk, "Real Belizean Food: Building Local Identity in the Transnational Caribbean", American Anthropologist, New Series101, no. 2 (1999): 244255. Guansheng Ma, "Food, eating behavior, and culture in Chinese society", Journal of Ethnic Foods 2(2015): 195-199. David. Y. H. Wu y Sidney C. H. Cheung, "Introduction: The Globalization of Chinese Food and Cuisine: Markers and Breakers of Cultural Barriers", en The Globalization of Chinese Food, ed. por David. Y. H. Wu y Sidney C. H. Cheung, (Londres: University of Hawai'i Press, 2002), 1-18.

13 Sandra Cabrera, "Marketing gastronómico. La experiencia de convertir el momento del consumo en un recuerdo memorable", Cuaderno. Centro de Estudios en Diseño y Comunicación 45 (2013): 165-174. 
dificulta que las mercancías se distingan entre sí, por eso suele recurrirse a la idea de biografía cultural para establecer su valor de acuerdo con la identidad de los autores o creadores. ${ }^{14}$ La visión biográfica de la comida está detrás de la creación de recetarios y libros de cocina, aquellos que dotan a ciertos platillos de la representación nacional china, dejando a otros fuera de la misma. Esta distinción conduce a otra que contrapone una supuesta alta cocina frente a otra considerada de baja calidad. Ese mismo fenómeno sucede en otros países como India, como lo indica Appadurai, ${ }^{15}$ quien sitúa la globalización de distintas gastronomías, entre ellas la china, como una posibilidad de que las élites locales muestren su poder económico y simbólico al hacer ostentación de su cosmopolitismo consumiendo ingredientes y platos de otros lugares.

Junto a las valoraciones sociales y simbólicas respecto de la comida china del Soconusco, también se toman en cuenta discusiones teóricas referidas a los procesos de globalización cultural de la propia gastronomía china, mismos que han generado distinciones locales y regionales, como ocurre en el caso de Tapachula. ${ }^{16}$ Por su parte, el marketing de experiencias memorables gastronómicas refiere un tipo de mercadotecnia en el que el cliente cuenta con expectativas únicas y memorables gracias a la construcción de nexos emocionales y sensoriales a través de la comida. ${ }^{17}$

Las anteriores referencias ayudan a contextualizar el objetivo del presente artículo, que es mostrar las diferentes representaciones sociales construidas en torno a la comida ofrecida en los negocios restauranteros de la ciudad. Estas representaciones sociales son entendidas como "estructuras preformadas a partir de las cuales se interpreta [...], la realidad”, al mismo

14 Kopytoff, "La biografía cultural de las cosas", 89-124.

15 Arjun Appadurai, "How to Make a National Cuisine: Cookbooks in Contemporary India”, Comparative Study of Society and History 30, no. 1 (1988): 3-24.

16 Wilk, "Real Belizean Food", 244-255. Ma, "Food, eating behavior, and culture”, 195-199. Wu y Cheung, "Introduction: The Globalization of Chinese Food $1-18$.

17 Cabrera, “Marketing gastronómico”, 165-174. 
tiempo que "intervienen en su elaboración". ${ }^{18}$ Esta circunstancia ratifica el parcial uso de los referentes culturales y nacionales y que, en este caso, se despliega en la representación de los procesos de inmigración procedentes de un mismo origen nacional y un destino de asentamiento común. La disociación se expresa en los discursos, con especial énfasis en los elaborados por los descendientes de la primera inmigración china que han sentido cuestionado el atributo de singularidad china en la costa chiapaneca.

En cuanto a la metodología, esta investigación se basa en entrevistas realizadas a más de cien descendientes de chinos en Tapachula, Escuintla y Tuxtla Gutiérrez durante diez años (2007-2014 y 2017-2019), así como entrevistas con personas vinculadas a comercios de comida china, al igual que a sus comensales. Ellos, como nacidos en México, hablan perfectamente castellano, caso contrario de los nuevos inmigrantes, quienes tienen dificultades con el idioma, además de que no resulta fácil convencerlos de entablar un diálogo y, mucho menos, realizar una entrevista. Asimismo, se han empleado técnicas etnográficas de observación participante, al consumir en los restaurantes de Tapachula, tanto los considerados tradicionales como los de reciente creación. Por último, también se han utilizado fuentes primarias y secundarias, con especial énfasis en las que construyen discursos sobre la gastronomía china en Chiapas.

\section{Comida china como fenómeno global}

La comida china servida en los considerados restaurantes tradicionales de Tapachula es observada como inalterable, es decir, se sostiene su permanencia gracias a la herencia cultural y familiar. Se trata de un exaltado legado transmitido por generaciones que, sin embargo, ha asimilado durante décadas ingredientes y métodos provenientes de otras tradiciones culinarias locales y foráneas. Si en un principio fue determinante la influencia de la cocina china de San Francisco, las incorporaciones no han cesado, como también ocurre en la China continental y en Taiwán. ${ }^{19}$ Los

18 Tomás Ibáñez, Ideologías de la vida cotidiana. Psicología de las representaciones sociales (Barcelona: Editorial Sendai, 1988), 36.

19 Wu y Cheung, "Introduction: The Globalization of Chinese Food”, 1-18. 
platos se ofrecen con técnicas mercadológicas para complacer a los clientes y, también, para captar turistas que arriban a la región. Son platillos representativos de la considerada tradición china, aunque su elaboración sea resultado de las conexiones transnacionales. ${ }^{20}$ De hecho, no es posible homogeneizar la oferta gastronómica china en su lugar de origen debido a la diversidad cultural del país, al mismo tiempo que muchos productos utilizados proceden de intercambios comerciales de largo aliento histórico, así como ocurre con las recetas y los métodos para preparar los platos. ${ }^{21}$ Esto contrasta con la visión de la comida como esencial de un territorio o grupo humano. ${ }^{22}$

Durante el siglo XXI, además del crecimiento acelerado de la economía china a nivel planetario, hay que destacar que los procesos de "globalización cultural" 23 han propiciado que distintas cocinas regionales de china hayan tenido una expansión planetaria. Una circulación gastronómica que facilita hablar de cocina cantonesa, macanesa, taiwanesa, pekinesa o hongkonesa, por citar algunos ejemplos. En la misma línea, pero aplicado a ciertos platillos, se encuentra el chop suey. Con origen en la cocina china de San Francisco (Estados Unidos), este plato se ha diversificado y popularizado alrededor del mundo. Se trata de una representación mundial de lo chino, aunque no tenga su origen en dicho territorio. Incluso en Estados Unidos hay más restaurantes de comida china que la suma de sucursales de McDonals y Burger King. ${ }^{24}$

20 Ulf Hannerz, Conexiones transnacionales. Cultura, gente, lugares (Valencia: Universitat de Valencia, 1998).

21 Wu y Cheung, "Introduction: The Globalization of Chinese Food”, 4.

22 Marina Tomic et al., "Consumers attitudes towards ethnic food consumption", Journal of Central European Agriculture 1, no. 2 (2018): 349-367.

23 Beck, ¿Qué es la globalización?, 71.

24 Emily Ma y Su Chen, "Western and Asian Customers' Perception Towards Chinese Restaurants in the United States", Journal of Quality Assurance in Hospitality $\sigma^{\circ}$ Tourism 12 (2011): 121-139. En Estados Unidos, los restaurantes de comida china han sido clasificados a través de tres características: 1) restaurantes dentro de los barrios chinos que ofrecen cocina china considerada "auténtica" y dirigida, principalmente, a comensales chinos; 2) restaurantes fuera de los barrios chinos y que atienden, sobre todo, a clientes estadounidenses; 3 ) restaurantes de unidad múltiple, aparecidos a finales de la década de 1970, y que son considerados de comida rápida (cfr. Ma y Chen, 123). 
Estas transformaciones del "ecúmene global" 25 son observables en la producción de alimentos, en su distribución y en las maneras de mesa, pero también en los negocios y consumos de los habitantes de la China continental. Los cambios son visibles en los nuevos emigrantes que salen de China hacia distintos países del orbe. ${ }^{26}$ De esta forma, la significación de las cocinas generadas por emigrantes chinos, tratadas como étnicas en otros países, forma parte de procesos históricos donde también se incluyen los migratorios. Dichos procesos generan representaciones sociales de la gastronomía en clave homogénea o heterogénea. Pueden ser homogéneas porque se las dota de una estructura cultural uniforme aunque, en realidad, sean el resultado de una diversidad interna y del cosmopolitismo de la propia gastronomía china en el mundo. ${ }^{27} \mathrm{Y}$ heterogéneas por las diferencias intrínsecas, además de poner en juego las prácticas de los actores en la cocina, así como los significados que los consumidores locales otorgan a dicha comida. ${ }^{28}$ En suma, son los comensales los que, con su capacidad de agencia y comunicación, otorgan valor a los platillos y crean hábitos alimenticios al consumirlos. Estos mismos consumidores pueden "volver criolla" la cocina de una región de China en territorios ajenos al país de origen. Tal circunstancia integra aquella práctica culinaria a imaginarios identitarios gastronómicos; una parte de ese "culto al patrimonio cultural”29 local sucede en el Soconusco y su ciudad capital, Tapachula.

\section{Identidad comestible en los restaurantes "tradicionales"}

El hecho de que la comida china sea un referente identitario en Tapachula es resultado de procesos locales y globales que convergen para

25 Hannerz, Conexiones transnacionales.

26 Wu y Cheung, "Introduction: The Globalization of Chinese Food1-18.

27 Wilk, "Real Belizean Foo", 244-255. Wu y Cheung, "Introduction: The Globalization of Chinese Food", 1-18.

28 Wilk, "Real Belizean Food", 248.

29 John L. Comaroff y Jean Comaroff, Etnicidad S.A. (Madrid: Katz Editores, 2011), 33. 
convertir una migración histórica en parte de la tradición cultural. Este supuesto ha sido convertido en discurso a través de los medios de comunicación y las políticas culturales regionales. De esta forma, la construcción de un imaginario alrededor de ella impacta con mayor fuerza para determinar su condición de realidad; ${ }^{30}$ lo convierte en un discurso valorado y devenido en patrimonio sin atisbos de crítica. ${ }^{31}$

Los descendientes de chinos asentados en otros países, como ocurre en el Soconusco, pueden defender su comida desde una perspectiva local. En consecuencia, el arribo de nuevos inmigrantes observados y considerados en muchas ocasiones como foráneos trastoca la forma de representar la sinidad en la Costa de Chiapas, donde juega un papel la defensa de la gastronomía china como legado cultural y contra la poderosa intromisión de la otredad china del presente. ${ }^{32}$ Así, la identidad cultural o étnica, convertida en "industria", ${ }^{33}$ en mercancía, ${ }^{34}$ según lo han expresado John y Jean Comaroff, también muestra nuevas divisiones en el seno de aquellos grupos humanos que compartirían vínculos culturales, ${ }^{35}$ como con claridad se aprecia en el caso aquí estudiado.

En tal sentido, los restaurantes consolidados en el Soconusco, administrados por descendientes chinos, apelan a la sinidad de su comida apoyándose en la recuperación de narrativas sobre la inmigración de sus ancestros a la región, así como en las aportaciones realizadas en la costa de Chiapas a lo largo del siglo XX. Ello justifica la creación de platillos chinos locales, que son considerados parte del patrimonio gastronómico regional por ser

30 Hannerz, Conexiones transnacionales.

31 Isabel Villaseñor Alonso y Emiliano Zolla Márquez, "Del patrimonio cultural inmaterial o la patrimonialización de la cultura”, Cultura y representaciones sociales 6, no. 12 (2012): 75-101.

32 Wilk, "Real Belizean Food", 244-255.

33 Comaroff y Comaroff, Etnicidad S.A., 45.

34 Ibíd., 9.

35 Ibíd., 220. 
elaborados en negocios de larga trayectoria histórica. ${ }^{36}$ De esta manera, en la ciudad de Tapachula se ofrece una amplia oferta restaurantera, en la que establecimientos tan conocidos como el Long Yin, el Confucio, el Men Cheng y el Kam Long se asumen como representantes de la auténtica comida china, incluso con referencias a sus vínculos con el estilo cantonés. En el restaurante Long Yin se afirma lo siguiente:

Utilizamos ingredientes y productos de primera calidad como verduras, camarones, pollo y carnes frescos, debidamente seleccionados para ofrecer platillos de la más alta calidad. Ofrecemos el mejor ambiente y el mejor sabor para que usted disfrute de su reunión, cumpleaños o comida de negocios, agasajando su paladar y el de sus acompañantes ya que nuestros platillos son preparados al momento con ingredientes frescos y naturales. Acuda con nosotros, en el Restaurant Long-Yin lo atenderemos como usted se merece con platillos exquisitos del país milenario que ha compartido su cocina tradicional en todo el mundo. ${ }^{37}$

Al mismo tiempo, las cartas de alimentos suelen ser amplias y con platos reconocibles en cualquier lugar del mundo a partir de la tradición culinaria china establecida en California, como puede ser el chop suey. Igualmente, incluyen ingredientes que no están presentes en los negocios de comida rápida, como el pato. También, en estos restaurantes se enfatiza que su comida está ligada a tradiciones originadas en China, como lo refiere el restaurante Confucio al aludir al Lam-Lam: "Durante las guerras chinas, familiares y amigos compartían sus víveres alrededor de una olla hirviendo. Así nace el Lam-Lam. En Confucio lo recreamos con ingredientes gourmet, logrando una experiencia y atmósfera únicas, en la tradición milenaria" ${ }^{38}$

36 Entrevista a Annie Mack, Tapachula, 25 de octubre de 2017; entrevista a Carlos Juan Chan, Tapachula, 11 de diciembre de 2017; entrevista a Hoilyg Maithe Cinco, Tuxtla Gutiérrez, 5 de noviembre de 2017; entrevista a Gilberto Liy Pérez, Tapachula, 09 de octubre de 2017; entrevista a Guillermo Chong Chiu, Tapachula, 25 de octubre de 2017.

37 Restaurant Long-Yin, "Presentación”, fecha de recuperación última modificación 18 octubre, 2021, link: https://restaurantlong-yinchinaweb.miadn.mx/

38 Confucio Comida China. "Menú del restaurante Confucio". Facebook. Agosto 22, 2021. https://www.facebook.com/Confucio8/photos/pcb.190472737636 $5906 / 1904727273032583$ 
De esta manera, la comida promovida en dichos restaurantes históricos se vincula con una región china, en concreto la provincia de Cantón, de donde provenían la mayoría de inmigrantes históricos asentados en Chiapas. Con respecto a la comida procedente de tal provincia, Ma considera que tiene nítidos usos sociales y rituales. ${ }^{39}$ Por ello es común en desayunos y cenas familiares, además de en otros ámbitos que crean amistades o establecen intercambios de negocios. A lo anterior se le denomina entre los chinos el guanxi; una forma de establecer círculos de confianza y reciprocidad en las relaciones interpersonales..$^{40}$ Por lo que respecta a Tapachula, las cafeterías chinas en la primera mitad del siglo XX fueron centros de reunión de los inmigrantes y sus descendientes; lugares para establecer amistades y alianzas entre familias. ${ }^{41}$ En relación con lo anterior, las actuales grandes porciones de los platillos chinos en los restaurantes tapachultecos recuerdan la forma de compartir varios platos entre los comensales. Esta es la presentación de la comida y la forma de consumir preferida por los clientes habituales, entre los que se encuentran familias de descendientes de chinos, la cual también se adapta a los cambios contemporáneos vinculados a los gustos locales, como lo manifestó Annie Mack, administradora del restaurante Kam Long en Tapachula:

Mis comensales son gente mayor, no gente joven, claro cuando vienen con familias vienen de todo, pero la mayoría son tapachultecos mayores. Los miembros de la comunidad china vienen aquí, al menos en Tapachula se consume más la comida cantonesa. La mayoría de los chinos que emigraron acá, eran de Cantón, fueron enseñando a los de aquí a comer la comida cantonesa. Como había muchos chinos de Cantón, se fue difundiendo la comida, nos hemos ido adecuando a los gustos de la gente de acá, porque ha cambiado el gusto con cada época [...]. Hemos modificado salsas, condimentos y sabores. Por ejemplo, a los rabioles fritos no les poníamos mayonesa, pero la gente nos pedía con mayonesa, entonces se le tuvo que poner porque así lo pedían. ${ }^{42}$

$39 \mathrm{Ma}$, "Food, eating behavior, and culture", 195.

40 Luciana Denardi, "Reflexiones Metodológicas sobre Investigaciones Etnográficas con 'Chinos' y 'Taiwaneses' en Argentina", Asian Journal of Latin American Studies 31, no. 2 (2018): 41-61.

41 Entrevista a Gilberto Liy Pérez, Tapachula, 09 de octubre de 2017; entrevista a Joaquín Ricardo Chiu, Tapachula, 11 de octubre de 2017.

42 Entrevista a Annie Mack, Tapachula, 25 de octubre de 2017. 
Igualmente, comer en un restaurante cantonés en el interior de China representa un elevado estatus social, ${ }^{43}$ algo similar podría replicarse en los restaurantes consolidados en Tapachula. Es decir, ir a dichos establecimientos implica una distinción por estatus de los consumidores ${ }^{44}$ al ser sus precios poco accesibles para todos los clientes. Estos establecimientos, gracias a sus biografías culturales, han edificado una imagen de marca que enfatiza que los consumidores comen la auténtica comida china. Este hecho es acompañado por la escenificación del espacio ocupado por el restaurante, que dota de atributos singulares la experiencia gastronómica, pues involucra los sentidos de los clientes a través de una memoria histórica positiva. ${ }^{45}$

De tal manera, los dueños de estos negocios han construido relaciones sociales con clientes asiduos a lo largo de los años; este nexo ha facilitado la generación de sentimientos empáticos al convertirse la oferta gastronómica en una evocación emocional. Lo expresado facilita que la oferta gastronómica de los restaurantes sea aprehendida por los clientes como parte de un patrimonio cultural local y reconocible; una parte de la historia del Soconusco y de Tapachula. Hace parte del testimonio vivo de una época pasada que está acompañado por el escenario de los recuerdos locales representado a través de la arquitectura ${ }^{46}$ y la ambientación de los negocios. ${ }^{47}$

La creación o transformación de platillos en dichos restaurantes está destinada a ilustrar una experiencia memorable en los consumidores. Por ello, ese grupo de restauranteros mantiene los presupuestos de la pureza

$43 \mathrm{Ma}$, "Food, eating behavior, and culture", 195-199.

44 Pierre Bourdieu, La distinción. Criterio y bases sociales del gusto (México: Taurus, 1988).

45 Michel Rochat, Marketing y Gestión de la Restauración (Barcelona, Gestión 2000, 2000), 96.

46 Josep Muntañola Thornberg, La arquitectura como lugar (Barcelona: Universitat Politècnica de Catalunya, 1974).

47 Cabrera, “Marketing gastronómico”, 165-174. 
e higiene ritual de su comida. ${ }^{48}$ Un paso en falso los insertaría dentro del grupo conformado por los nuevos negocios gastronómicos de Tapachula, abiertos por los inmigrantes chinos llegados durante el presente siglo.

En algunos casos, los descendientes de chinos del Soconusco que administran restaurantes tradicionales, con el objetivo de ampliar su biografía cultural y marketing gastronómico, han sentido la necesidad de viajar a China para tomar cursos de gastronomía de ciertas regiones. Su propósito ha sido lograr una hibridación gastronómica al aprender sobre la preparación, condimentación y presentación de recetas. ${ }^{49}$ Estas acciones dan un nuevo valor a los restaurantes a ojos de los consumidores, aunque representen un impacto en el costo de los alimentos. De este modo, la satisfacción de expectativas alimentarias por parte de los comensales se reafirma con un marketing que garantiza su prestigio, apoyado en dos facetas primordiales, una sustentada en la calidad de los productos de su oferta gastronómica y, la otra, amparada en su higiene y cuidado para manipular los alimentos. Ello aparece reforzado por la idea de que la comida china de la región forma parte de una tradición cultural transmitida por generaciones y con un sello familiar:

En el Long Yin y Confucio son familias de restaurantes. Siguen manteniendo el sello de restaurantes tradicionales, al heredar la comida china en su familia, por cada descendiente; recetas, ya sabe, toque familiar. Hay un sello familiar, con cada apellido. Nosotros los Mack tenemos uno. ${ }^{50}$

Como contrapunto a lo expresado, hay que señalar que el compartido origen nacional con los nuevos inmigrantes chinos no es impedimento para establecer distinciones culturales y simbólicas con ellos y sus negocios. El estatus social adquirido por los descendientes de chinos asentados en Chiapas también se utiliza como diferenciación. A ello hay que añadir otro distanciamiento, en este caso establecido por los inmigrantes recién llegados, quienes utilizan parámetros biológicos para identificar a

48 Mary Douglas, Pureza y peligro. Un análisis de los aspectos de contaminación y tabú (Madrid: Siglo XXI, 1991).

49 Entrevista a Alberto Joo Vera, Tuxtla Gutiérrez, el 27 de noviembre de 2017.

50 Entrevista a Annie Mack, Tapachula, 25 de octubre de 2017. 
los descendientes de chinos como mezclados - mestizos según muchos de los descendientes de chinos-. Se traza así una frontera forjada en la sangre y en una idea de autenticidad y pureza leída con parámetros raciales.

\section{Nueva oferta gastronómica como confrontación local}

Con la llegada de nuevos inmigrantes ha emergido un debate donde la tradición culinaria china, convertida en la auténtica por ancestral, ${ }^{51}$ se confronta a la nueva oferta de los chinos recién llegados a Tapachula. La autenticidad es una definición cultural enmarcada por atributos construidos y donde es imposible obviar la subjetividad. ${ }^{52}$ En el caso de la comida china, otorgarle ciertas cualidades a la preparada por los descendientes de chinos, por su trasfondo familiar y generacional, es señalado por un comensal de Tapachula, descendiente chino de segunda generación como un rasgo deseable:

Si quieres comer buena comida, te recomendaría algunos buenos restaurantes de comida china. Ahí va sesgado porque yo te recomendaría donde yo como, que son mis amigos, y hay muchos restaurantes nuevos que no como ahí, porque ellos han hecho comida para la localidad. Hoy en día en todo el mundo la comida china tiene dos vertientes, una, la comida económica, abundante y barata, y la otra comida buena, tradicional. Entones yo voy a restaurantes, incluso me conocen, y me cocinan especial para mí. Me gusta el chao mein, me lo hacen, pero eso no te lo van a servir a ti. Pero entonces para qué te lo recomiendo sino te van a servir a ti, lo que van servirme a mí. ${ }^{53}$

De esta manera, la comida china tiene una doble vara para mesurarse. $\mathrm{Si}$ se asocia a las migraciones del siglo $\mathrm{XX}$, se revalora por ser parte del patrimonio cultural del Soconusco; por el contrario, si se relaciona con la reciente migración china adquiere una connotación negativa por consi-

51 Rincón Zárate, "El posicionamiento socio-espacial”.

52 Bryan Spooner, "Tejedores y comerciantes: La autenticidad de una alfombra oriental", en La vida social de las cosas. Perspectiva cultural de las mercancías, ed. Arjun Appadurai, (México: CONACULTA/Editorial Grijalbo, 1991), 409-448. 53 Entrevista a Enrique Mak, Tapachula, 18 de octubre de 2017. 
derarse una comida chatarra y popular, sin ningún sesgo de autenticidad, aunque proceda del mismo país y sea preparada por chinos. Mientras los descendientes de chinos son parte de la representación patrimonial del pasado regional, los recién llegados entrañan todo lo contrario, aunque tengan el mismo origen nacional. Es común, entonces, que se establezcan distinciones peyorativas hacia los nuevos restaurantes de comida exprés, incluso atribuyéndoles un origen asiático, pero no chino. ${ }^{54}$

El primer restaurante creado con la modalidad que utilizan los nuevos inmigrantes fue el Bambú, céntrico establecimiento cuyo propietario es un descendiente chino que privilegia la entrega a domicilio y la comida para llevar debido al reducido espacio del local. Los nuevos restaurantes ofrecen platillos recurrentes como el arroz blanco y frito, los ravioles, el chow mein o el chop suey, aunque suelen tener varias combinaciones de guisos en un mismo plato. Además de especificar, en su mayoría, el carácter cantonés de su cocina, destacan por la abundancia de las raciones y la asequibilidad de sus precios. Lo anterior los convierte en una opción para todo tipo de consumidores. Ello está vinculado al bajo costo de preparar alimentos en grandes cantidades, así como a utilizar aceites y condimentos más económicos. Un ejemplo de lo expuesto es la adquisición de salsas comercializadas, como las que tienen como base el jengibre o el ostión; en contraposición con la elaboración efectuada en los restaurantes tradicionales donde sí las preparan. Al mismo tiempo los locales, que no suelen ser espaciosos, tienen referencias a China en su decoración, como los característicos globos para sus lámparas o dragones pegados en las paredes.

Frente a esa oferta gastronómica se confronta la comida elaborada por los descendientes de chinos, elevada a patrimonial a través del discurso que le otorga profundidad histórica, una situación que refuerza la idea de Tapachula como ciudad abierta ${ }^{55}$ :

la comida china para los tapachultecos que comercializan o consumen estos productos, tiene una biografía cultural, con una fuerte valorización de quién hace esos productos chinos, [...] personas trabajadoras, con un gran

54 Entrevista a Santiago Martínez Junco, Tapachula, 1 septiembre de 2017. 55 Rincón Zárate, “El posicionamiento socio-espacial”, 108. 
legado cultural milenario, el resguardo de la receta familiar y una historia familiar migratoria detrás de la preparación de los platillos, retomando el valor simbólico a estos platillos por su antigüedad y autenticidad. ${ }^{56}$

Los nuevos restaurantes chinos aprovechan la globalización cultural de la comida china para ofrecer otras opciones gastronómicas que, en muchos casos, proceden de experiencias migratorias en otros lugares del planeta. Esta adaptación también remite a los productos de los que disponen y a las demandas de los comensales con menor poder adquisitivo. Igualmente, como ya se mencionó, los chinos llegados recientemente dispensan comida rápida frente a la comida a la carta de los antiguos restaurantes. Esta circunstancia abarata el precio pagado por el consumidor, con lo que se establece una nítida competencia económica que tiende a ser contrarrestada por la idea de calidad y, sobre todo, de legitimidad que otorga la tradición culinaria. Esta es una afirmación secundada por los comentarios negativos que redimen en el presente los prejuicios históricos a través de aseveraciones sobre la dudosa condición higiénica de los nuevos establecimientos de comida china, tal como la prensa local de los últimos años ha manifestado. ${ }^{57}$ Esto representa un eslabón más de la extensa historia de prejuicios contra la inmigración china, donde el dispendio de alimentos se erige en elemento para la recepción de todas las opiniones xenófobas. ${ }^{58}$ Aunque no es ninguna novedad en el país y en otros lugares de América Latina, ${ }^{59}$ en las últimas fechas se ha extendido a otros fenómenos internacionales como los relacionados con la propagación del coronavirus, ${ }^{60}$ en especial a través del ingreso comercial de productos asiáticos. ${ }^{61}$

56 Ibíd., 156.

57 Rincón Zárate, “El posicionamiento socio-espacial”, 150.

58 Lisbona Guillén, Alli donde lleguen, 137.

59 Patricia Palma y Maria Montt Strabucchi, "Chinese Business in Latin America and the Caribbean: A Historical Overview", Journal of Evolutionary Studies in Business 4, no. 2 (2019): 198-200.

60 Jun He et al., "Discrimination and Social Exclusion in the Outbreak of COVID-19", International Journal of Environmental Research and Public Health 17, no. 2933 (2020): 1-4. Véase la caricatura referida al coronavirus y los chinos: “\%\#\% CHINOS...” en El Orbe, Tapachula, 23 de mayo de 2020. Obtenida el 2505-2020: https://elorbe.com/seccion-politica/cartones/2020/05/23/chinos.html 61 Sergio García, "Preocupa ingreso de productos chinos”, en NVINoticias, 03 de marzo de 2020. Obtenido el 10-03-2020 en: https://www.nvinoticias.com/ nota/139436/preocupa-ingreso-de-productos-chinos 
Las emergentes distinciones entre descendientes y nuevos inmigrantes, ejemplificadas con la comida china, no deben considerarse como totalmente novedosas. De hecho, dentro de los descendientes de inmigrantes ya se habían hecho presentes a la hora de definirse como comunidad china y en las discrepancias entre las asociaciones de danzas existentes en el Soconusco. ${ }^{62}$ En estos diferendos, entre otros factores, aparece el de la sangre como elemento perturbador de los grupos dancísticos al no contar con muchos miembros de ascendencia china.

$\mathrm{Al}$ mismo tiempo, la relación entre los descendientes de chinos y los nuevos inmigrantes en la región no ha sido fluida, al menos en sus inicios, puesto que se erigen en competencia comercial. Carlos Juan, cuyos padres eran de Cantón y Macao, y que es dueño del restaurante Long Yin en Tapachula, confirma la escasa relación:

He notado la presencia de nuevos migrantes chinos. [Entre] estos tipos de migrantes chinos no hay mucha comunicación con la Comunidad China. Ellos sólo se dedican a trabajar, su mentalidad es trabajar y trabajar más. Ellos vienen de Kuan Chau, vienen hace ocho, nueve años, algunos desde hace doce ańos. Puedo hablar con los que hablan cantonés, con los de mandarín casi no. Mucha gente que vino habla el mandarín, no hay mucha comunicación con ellos, son muy apartados. Ellos se dedican a vender comida, es lo único que saben, el oficio de vender comida, comerciantes algunos, pero es comida buffet. Los restaurantes longevos chinos somos diferentes a ellos. ${ }^{63}$

Así, el arribo de estos inmigrantes y sus negocios es confrontada a través de dos vertientes que, en ningún caso, toman de referente su origen común chino y que podría ubicarlos como parte de esa imaginada comunidad basada en formar parte del mismo colectivo nacional. Por el contrario, su procedencia no ha influido para mesurar la confrontación de su presencia en la ciudad. Las arremetidas hacia los nuevos migrantes se han centrado en su marcado aislamiento social y escaso interés en tener contacto con los descendientes de chinos, por una parte, mientras que, por otra, los reclamos se han expuesto atribuyendo falta de calidad del producto alimenticio ofrecido en sus establecimientos. Estas percepciones son se-

62 Lisbona Guillén, "Danzas como tradición”, 9-28.

63 Entrevista a Carlos Juan, Tapachula, 11 de diciembre de 2017. 
cundadas por ciertos consumidores a causa de los prejuicios siempre presentes hacia los inmigrantes. Los nuevos inmigrantes chinos llegan con un capital económico para competir de forma abierta con los restaurantes tradicionales de comida regentados por descendientes de chinos; es decir, no cumplen, entre otras cosas, con la existencia de una biografía cultural que dote de legitimidad a sus establecimientos. Este hecho es ratificado por la carente ambientación china de los establecimientos, más allá de los letreros o cartas del negocio. Ello provoca que no se vincule su oferta gastronómica con la comida china considerada tradicional; un discurso extendido por los descendientes de chinos que no desean ser influenciados por los nuevos restauranteros porque podría significarles la pérdida de su sello de identidad dentro del patrimonio cultural del Soconusco. Es decir, para un restaurante con el referente de tradicional ser vinculado con la nueva oferta gastronómica implica perder su biografía cultural y el valor simbólico atribuido a su comida. Esto refuerza la distinción social establecida a través del consumo alimentario y constituye una neofobia alimentaria aplicada hacia los nuevos negocios chinos. ${ }^{64}$

\section{Reflexiones finales}

Si los primeros inmigrantes llegados mayormente a principios del siglo XX organizaron sus actividades a través del Kuo Ming Tang, pensado como un lugar de reunión y de ayuda mutua entre los inmigrantes, así como una forma de mantener los vínculos con su país de origen, tal situación sufrió posteriores quiebres generacionales. Esta ruptura fue expuesta esquemáticamente en las entrevistas efectuadas a inmigrantes originales en Tapachula.$^{65}$ Ello también es visible en otros países del continente americano, ${ }^{66} \mathrm{y}$ es apreciable en la imaginación de una comunidad china consolidada pero inexistente, es decir, los intentos de institucionalizar una

64 Tomic et al., 2018. "Consumers attitudes", 349-367.

65 Rebeca Lau, "Memories of origins/Origins of memories. The colletive memory of the Chinese community in Tapachula, Chiapas, Mexico" (Tesis de Maestría. The University of British Columbia, 2003), 21-44.

66 Alejandro Grimson, Gustavo NG y Luciana Denardi, "Las organizaciones de inmigrantes chinos en Argentina”, Migración y desarrollo 14, no. 26 (2016): 4243. 
organización convertida en referente comunal, como las instituciones históricas, han tenido poco éxito. Esta situación puede equipararse a lo que ocurre con otros colectivos descendientes de migraciones históricas, como los libaneses, turcos o japoneses en el Soconusco, quienes también tienen dificultades para mostrarse como una "comunidad integrada" ${ }^{67}$

En relación con lo anterior, los descendientes de inmigrantes chinos en Tapachula han utilizado elementos culturales o recuerdos de los lugares de origen de sus familiares para dotarse de una identidad singular que les permitiera construir una cohesión social china. No obstante, en muchas ocasiones, esta pretensión resulta en un hermanamiento imposible, ${ }^{68}$ un simulacro en el sentido de Jean Braudrillard. ${ }^{69} \mathrm{La}$ inviabilidad de tal proyecto no ha impedido utilizar recursos culturales tangibles, como la comida china, convertida en el referente principal de la inmigración china en Chiapas.

La dificultad para urdir una comunidad china integrada y reconocible por parte de los descendientes de chinos, al mismo tiempo que los elementos destacados de la sinidad en la costa chiapaneca, como la comi$\mathrm{d} a$, se enfrentan en los años recientes del siglo XXI al arribo de nuevos inmigrantes. Este fenómeno ha llamado la atención a los medios de comunicación ${ }^{70} \mathrm{y}$ también al medio académico a través de incipientes investigaciones. Los nuevos inmigrantes resultan difíciles de idear para los descendientes de chinos chiapanecos, a pesar de compartir el origen común

67 Martin Yoshio Cruz Nakamura, "Más allá del mar: la comunidad Nikkei de México, caso Soconusco de Chiapas y cuidad de México" (Tesis de Doctorado, Universidad de Ciencias y Artes de Chiapas, 2020), 109-110.

68 Manuel Delgado Ruiz, Diversitat i integració. Lògica i dinámica de les identitats a Catalunya (Barcelona: Editorial Empúries, 1998), 82-85.

69 Jean Braudrillard, Cultura y Simulacro (Barcelona: Editorial Kairós, 1987).

70 Véase, por ejemplo, el reportaje signado por Zenyazen Flores, "México vive segunda oleada de inmigrantes provenientes de China”, en El Financiero, México, 03 de marzo de 2014. Obtenido el 30-06-2020 en: https://www.elfinanciero.com. $\mathrm{mx} /$ economia/mexico-vive-segunda-oleada-de-inmigrantes-provenientes-de-china 
de la China continental. De hecho, el arribo de nuevos inmigrantes, en especial a Tapachula, ha convertido al centro de la ciudad en un espacio debatido por ser ocupado por muchos de los nuevos negocios de comida de los inmigrantes recién llegados. La gastronomía china, convertida en la típica local y referente identitario regional, se ha visto hollada por los nuevos inmigrantes chinos que se incorporan a la oferta restaurantera como competencia de los establecimientos considerados tradicionales. De esta forma, si la comida china era un valor que parecía intocable como diacrítico de identificación de los descendientes chinos, a la vez que era su mayor aportación a la identidad regional, ahora evidencia que los elementos culturales no pueden considerarse como esenciales y, mucho menos, aprisionarse, dados los procesos transnacionales contemporáneos. ${ }^{71}$

El grupo de nuevos restauranteros responde de forma divergente a la representación de lo chino respecto de los descendientes de la primera inmigración. Estos últimos, no nacidos en China, acuden a lo chino como tradición familiar y resultado de la versión local de lo chino, como ocurre con descendientes de inmigrantes en otras latitudes del mundo. ${ }^{72}$ En esa dirección, existen elementos culturales, como las expresiones dancísticas y culinarias, pasadas por el tamiz chiapaneco. Estos referentes identitarios permiten imaginar la existencia grupal, para rememorar un origen común y cohesión social que en algún momento histórico pudo existir y que se cree reeditable. ${ }^{73}$ Sin embargo, conforman un "pacto fáustico" que puede acabar en la "autoparodia" "74 grupal o en una benigna simulación, alentada por mediadores culturales con el anhelo de hallar en las trasnochadas supervivencias, y en las fáciles cárceles de las raíces culturales, explicaciones para clasificar grupos humanos según su origen geográfico.

71 Hannerz, Conexiones transnacionales. Beck, ¿Qué es la globalización?.

72 William A. Douglass, "Factores que intervienen en la formación de la diáspora emigrante vasca al Nuevo Mundo”, en Los otros vascos. Las migraciones vascas en el siglo XX, comp. por F. Xavier Medina (Madrid: Editorial Fundamentos, 1997), 34.

73 Manuel Delgado Ruiz, Diversitat i integració, 82-85.

74 Comaroff, y Comaroff, Etnicidad S.A., 48. 
Diversas investigaciones han resaltado como el papel de la "sangre" se convierte en un factor de confianza para poder establecer relaciones con los nuevos inmigrantes chinos, ${ }^{75}$ sin embargo, esta certeza no resuelve uno de los principales aspectos del debate sobre los contactos entre descendientes de inmigraciones históricas y los recién llegados de la misma procedencia nacional. La investigadora Evelyn Hu-Dehart ejemplificó la multiplicidad de ideas que rodean la autopercepción de inmigrantes o de sus descendientes, en este caso a través de la presencia china en el Caribe:

Según las palabras del destacado historiador trinitario de origen chino Walton Look Lai, durante el curso de los 150 años de experiencia en el Caribe, los chinos han pasado de ser una "comunidad de inmigrantes extranjeros" a convertirse en una "minoría étnica nacional". La colega y especialista chino-trinitaria Christine Ho sostiene la tesis de que los chinos en las Indias Occidentales británicas se han criollizado o asimilado, mientras que el artista jamaiquino de origen afrochino, Albert Chong, describe su mezcla racial y étnica como un aspecto inseparable de ser jamaiquino. ${ }^{76}$

Estas aseveraciones divergentes, por un lado, explican las dificultades clasificatorias de los propios involucrados. Por otro lado, sin caer en el culturalismo redivivo actual que, como señala Arjun Appadurai, se ha instalado "como el principal escenario donde se representan fantasías de pureza, autenticidad, fronteras y seguridad", 77 es innegable que ciertos símbolos culturales se han convertido en referencias para el caso de los chinos y sus descendientes en Chiapas. Con certeza, la comida china es el aspecto más llamativo por trascender a un colectivo humano de inmigrantes y convertirse en bandera patrimonial del territorio de acogida. Esta legitimación fue otorgada por un pasado donde la comida china

75 Grimson, NG y Denardi, "Las organizaciones de”, 26-27.

76 Evelyn Hu-Dehart, "Los culíes, los tenderos y sus descendientes”, en Cuando oriente llegó a América. Contribuciones de inmigrantes chinos, japoneses y coreanos, ed. Banco Interamericano de Desarrollo (Washington: Banco Interamericano de Desarrollo, 2004), 18.

77 Arjun Appadurai, El rechazo de las minorias. Ensayo sobre la geografía de la furia. (Barcelona: Tusquets Editores, 2007), 38. 
ayuda al afianzamiento del patrimonio cultural de Tapachula como una reafirmación de su cosmopolitismo.

El carácter contingente y mudable de los hechos culturales, y el constante replanteamiento de los elementos que los configuran, es más visible gracias al arribo de nuevos inmigrantes chinos a la región del Soconusco. El supuesto carácter estático de la comida china, asentada y difundida como la tradicional en la costa chiapaneca, entra en confrontación con las ofertas de comida aportadas por estos nuevos inmigrantes. Situar a la comida china como la auténtica, por ser la realizada por los descendientes de chinos, degrada cualquier novedad, como la aportada por los inmigrantes recién llegados. Por consiguiente, la comida china ilustra y ejemplifica los conflictos por las definiciones y las representaciones de lo chino. En este debate, lo chino queda escindido en tramas sociales locales y globales para cuestionar la inmovilidad de las identidades culturales, al igual que las nacionales, al mismo tiempo que demuestra la capacidad de las representaciones sociales para orientar "las conductas y las relaciones". ${ }^{78}$

\section{Bibliografía citada}

Anderson, Benedict, Comunidades imaginadas. Reflexiones sobre el origen y la difusión del nacionalismo. México: Fondo de Cultura Económica, 1993.

Appadurai, Arjun. "How to Make a National Cuisine: Cookbooks in Contemporary India". Comparative Study of Society and History 30, no. 1 (1988): 3-24.

Appadurai, Arjun. El rechazo de las minorias. Ensayo sobre la geografía de la furia. Barcelona: Tusquets Editores, 1999.

Beck, Ulrich. ¿Qué es la globalización? Falacias del globalismo, respuestas a la globalización. Lanús: Paidós, 2008.

Bourdieu, Pierre. La distinción. Criterio y bases sociales del gusto. México: Taurus, 1988.

78 Denise Jodelet, "La representación social: fenómenos, concepto y teoría". en Psicología Social II. Pensamiento y vida social. Psicología social y problemas sociales, comp. Serge Moscovici (Barcelona: Paidós, 1986), 486. 
Braudrillard, Jean. Cultura y Simulacro. Barcelona: Editorial Kairós, 1987.

Cabrera, Sandra. "Marketing gastronómico. La experiencia de convertir el momento del consumo en un recuerdo memorable". Cuaderno. Centro de Estudios en Diseño y Comunicación 45 (2013): 165-174.

Comaroff, John L. y Jean Comaroff. Etnicidad S.A. Madrid: Katz Editores, 2011.

Cruz Nakamura, Martin Yoshio. "Más allá del mar: la comunidad Nikkei de México, caso Soconusco de Chiapas y cuidad de México". Tesis de Doctorado. Universidad de Ciencias y Artes de Chiapas, 2020.

Delgado Ruiz, Manuel. Diversitat i integració. Lògica i dinámica de les identitats a Catalunya. Barcelona: Editorial Empúries, 1998.

Denardi, Luciana. "Reflexiones Metodológicas sobre Investigaciones Etnográficas con 'Chinos' y 'Taiwaneses' en Argentina". Asian Journal of Latin American Studies 31, no. 2 (2018): 41-61.

Douglas, Mary. Pureza y peligro. Un análisis de los aspectos de contaminación y tabú. Madrid: Siglo XXI, 1991.

Douglass, William A. "Factores que intervienen en la formación de la diáspora emigrante vasca al Nuevo Mundo". En Los otros vascos. Las migraciones vascas en el siglo XX, compilador por $\mathrm{F}$. Xavier Medina, 27-49. Madrid: Editorial Fundamentos, 1997.

Glick Schiller, Nina. "Transmigrants and Nation States: Something Old and Something New in the U.S. Immigrant Experience”. En Handbook of International Migration: The American Experience, editado por C. Hirschman, P. Kasinitz y J. De Wind, 94-119. Nueva York: Russell Sage Foundation, 1999.

Grimson, Alejandro, Gustavo NG y Luciana Denardi. "Las organizaciones de inmigrantes chinos en Argentina”. Migración y desarrollo 14, no. 26 (2016): 25-73.

Hannerz, Ulf. Conexiones transnacionales. Cultura, gente, lugares. Valencia: Universitat de Valencia, 1998.

He, Jun, Leshui He, Wen Zhou, Xuanhua Niey Ming He. "Discrimination and Social Exclusion in the Outbreak of COVID-19". International Journal of Environmental Research and Public Health 17, no. 2933 (2020): 1-4. 
Hernández López, María del Jazmín. "El comercio chino en San Cristóbal de Las Casas, Chiapas: tendencias hacia la empresa familiar, el empresariado étnico y el sistema de redes familiares”. Tesis de Maestría en Antropología Social. Centro de Investigaciones y Estudios Superiores en Antropología Social, 2015.

Hu-Dehart, Evelyn. "Los culíes, los tenderos y sus descendientes". En Cuando orientellegó a América. Contribuciones de inmigrantes chinos, japoneses y coreanos, editado por Banco Interamericano de Desarrollo, 15-34. Washington: Banco Interamericano de Desarrollo, 2004.

Ibáñez, Tomás. Ideologias de la vida cotidiana. Psicología de las representaciones sociales. Barcelona: Editorial Sendai, 1988.

Jodelet, Denise. "La representación social: fenómenos, concepto y teoría”. En Psicología Social II. Pensamiento y vida social. Psicología social y problemas sociales, compilador por Serge Moscovici, 469-493. Barcelona: Paidós, 1986.

Kopytoff, Igor. "La biografía cultural de las cosas: La mercantilización como proceso". En La vida social de las cosas. Perspectiva cultural de las mercancias, editado por Arjun Appadurai, 89-124. México: CONACULTA/Editorial Grijalbo, 1991.

Lau, Rebeca. "Memories of origins/Origins of memories. The colletive memory of the Chinese community in Tapachula, Chiapas, Mexico". Tesis de Maestría. The University of British Columbia, 2003.

Lisbona Guillén, Miguel. Alli donde lleguen las olas del mar. Pasado y presente de los chinos en Chiapas. México: UNAM-PROIMMSE/ CONACULTA-CONECULTA, 2014.

Lisbona Guillén, Miguel. "Danzas como tradición y como disputa: la ilusión comunitaria china en el Soconusco chiapaneco". Peninsula X, no. 1 (2015): 9-28.

Ma, Emily, y Su Chen. 2011. "Western and Asian Customers' Perception Towards Chinese Restaurants in the United States". Journal of Quality Assurance in Hospitality $\sigma^{\circ}$ Tourism 12 (2011): 121-139.

Ma, Guansheng, "Food, eating behavior, and culture in Chinese society", Journal of Ethnic Foods 2 (2015): 195-199. 
Muntañola Thornberg, Josep. La arquitectura como lugar. Barcelona: Universitat Politècnica de Catalunya.

Palma, Patricia, y Maria Montt Strabucchi, "Chinese Business in Latin America and the Caribbean: A Historical Overview". Journal of Evolutionary Studies in Business 4, no. 2 (2019): 175-203.

Portes, Alejandro, Cristina Escobar y Alexandria Walton. "Organizaciones transnacionales de inmigrantes y desarrollo: un estudio comparativo”. Migración y Desarrollo 6 (2006): 3-44.

Rincón Zárate, Ulises. "El posicionamiento socio-espacial de los descendientes de inmigrantes chinos y japoneses en Tapachula, Chiapas". Tesis de Maestría. Centro de Investigaciones y Estudios Superiores en Antropología Social, 2019.

Rochat, Michel. Marketing y Gestión de la Restauración. Barcelona: Gestión 2000, 2000.

Spooner, Bryan. "Tejedores y comerciantes: La autenticidad de una alfombra oriental”. En La vida social de las cosas. Perspectiva cultural de las mercancías, editado por Arjun Appadurai, 409-448. México: CONACULTA/Editorial Grijalbo, 1991.

Tébar Arjona, Jesús. "Patrones espaciales de la diáspora china en el mundo, España y Madrid”. Historia Actual Online, no. 30 (2013): 89-103

Tomic, Marina, Kristiana Deronja, Milna Tudor Kalit y Željka Mesić. "Consumers attitudes towards ethnic food consumption". Journal of Central European Agriculture 1, no. 2 (2018): 349-367.

Villaseñor Alonso, Isabel, y Emiliano Zolla Márquez. "Del patrimonio cultural inmaterial o la patrimonialización de la cultura". Cultura $y$ representaciones sociales 6, no. 12 (2012): 75-101.

Wilk, Richard R. "Real Belizean Food": Building Local Identity in the Transnational Caribbean. American Antbropologist, New Series 101, no. 2 (1999): 244-255.

Wu, David. Y. H., y Sidney C. H. Cheung. "Introduction: The Globalization of Chinese Food and Cuisine: Markers and Breakers of Cultural Barriers". En The Globalization of Chinese Food, editado por David. Y. H. Wu y Sidney C. H. Cheung, 1-18. Londres: University of Hawai'i Press, 2002. 